\title{
Entre el prosperar colectivo de Ellas Hacen y la meritocracia individual del Hacemos Futuro Juntas
}

Between the collective prospering of Ellas Hacen (Women who do) and the individual meritocracy of Hacemos Futuro Juntas (We women make future together)

\section{Tatiana Marisel Pizarro}

CONICET - Universidad Nacional de San Juan, Argentina

\begin{abstract}
RESUMEN El objetivo de este artículo es el de presentar la evolución del concepto trabajo en la política social argentina "Ellas hacen" -que fomentaba la creación de cooperativas de mujeres trabajadoras para alcanzar su autonomía económica- hasta su unificación en el programa "Hacemos Futuro Juntas" -que instaba al desarrollo personal-. Para realizar este análisis fue preciso hacer un recorrido por las gestiones gubernamentales de Cristina Fernández de Kirchner (2017-2019) y de Mauricio Macri (2019-2020). De este modo, se problematizó en la manera en que esta política fue (re)definiendo al trabajo desde un pretendido enfoque de género, siendo que, en la evolución de ésta, en su diseño y su práctica omitían problemáticas relacionadas al mismo. Por lo que, fue preciso articularse a la perspectiva de los estudios de género a las políticas públicas. En este aspecto es en el que se manifiesta la importancia del paper, ya que se expone cómo el Estado exceptúa las singularidades de las mujeres en este tipo de medidas, lo que ayudaría a ahondar más aún las inequidades entre los géneros.
\end{abstract}

PALABRAS CLAVE Mujeres; estado; políticas sociales; feminización de la pobreza. 


\begin{abstract}
The objective of this article is to present the evolution of the concept 'work' in Argentine social policy from Ellas Hacen, which encouraged the creation of cooperatives of working women to achieve their economic autonomy, to its unification in the Hacemos Futuro Juntas policy, which urged personal development. To carry out this analysis, it was necessary to review the government efforts of Cristina Fernández de Kirchner (2017-2019) and of Mauricio Macri (2019-2020). Thus, the way in which this policy was (re) defining the concept work from a purported gender perspective was problematized, since in its evolution, in its design and practice, the policy omitted problems related to it. In this sense, it was necessary to articulate the perspective of gender studies with public policies. It is in this aspect that the importance of this paper is manifested, since it exposes how the State exempts the singularities of women in this type of policies, which would contribute to deepen inequalities between genders.
\end{abstract}

KEYWORDS Women; State; social politics; feminization of poverty.

\title{
Introducción
}

Durante los primeros quince años del siglo XXI, los programas de ajuste estructural enraizados en América Latina cambiaron el rumbo que tenían las políticas sociales hasta entonces, lo que hizo que el Estado dejara de ser el responsable de los mecanismos redistributivos que beneficiasen a todos/as los/as ciudadanos/as para segmentar su atención en grupos y proyectos particulares.

En el caso de América Latina, se impulsaron los Programas de Transferencias Condicionadas de ingreso (PTC) como la principal forma de intervención de los gobiernos para atender a la población en situación de pobreza, mediante la garantía de cierto nivel básico - en general mínimo- de ingresos monetarios (Rodríguez Enríquez, 2011). A raíz de los PTC, el paternalismo estatal determinó que la mejor forma de atenuar las situaciones de pobreza era la de satisfacer las necesidades básicas de la población en situación de vulnerabilidad socioeconómica a través de transferencias monetarias focalizadas y condicionadas ${ }^{1}$. En este sentido, se planteó la necesidad de tener un enfoque operativo ${ }^{2}$ que facilitase la observación de políticas públicas (Subirats, 2000).

1. El porqué de los programas de este tipo está dado en "la equidad de la eficiencia", en lo que se efectivizan los escasos recursos públicos invirtiéndolos donde es más relevante y necesaria su acción, lo que lleva a los gobiernos a reducir su gasto público general con el incremento a su vez del apoyo social.

2. De este enfoque se desprendan ocho componentes claves: la solución de un problema público (la política surge para resolver una demanda social reconocida políticamente como un problema 
Esto resultó de gran interés para observar, analizar y describir la manera en que se encuentra presente el trabajo en el Programa Ellas Hacen y su posterior devenir en el Programa Hacemos Futuro Juntas. Se considera a esta medida como icónica, ya que fue gestada en Argentina durante la década kirchnerista (2003-2015), momento histórico en el que se establecieron distintas políticas sociales que se construyeron sobre tres ejes: inclusión, universalidad y solidaridad.

Debido a esto, en este artículo, se busca problematizar en la manera en que esta política fue (re)definiendo al trabajo desde un pretendido enfoque de género, por lo que en estas páginas se hará hincapié en la evolución de esta medida, en su diseño y su práctica. En este aspecto es en el que se manifiesta la importancia de este escrito, ya que se intenta mostrar la manera en que el Estado exceptúa las singularidades de las mujeres en este tipo de medidas, lo que ayudaría a ahondar más aún las inequidades entre los géneros.

Para cumplir con el propósito planteado, se acopla la mirada de los estudios de género a las políticas públicas, por lo que las reflexiones que constituyen a este artículo devienen de un análisis teórico-crítico del programa mencionado.

Son tres las partes que componen este documento: en primer lugar, se enuncian las características de la ciudadanía de las mujeres en Latinoamérica y las particularidades de las políticas públicas en este contexto histórico-espacial, pero poniendo el foco en Argentina. Luego, se presenta el Programa Ellas Hacen y sus características principales: desarrollo de la política, creación de cooperativas, dictado de talleres de formación y las particularidades de las destinatarias. Finalmente, se muestran los cambios más significativos en términos de perspectiva de género que la política tuvo a partir del cambio de gestión gubernamental.

\section{Las mujeres y su ciudadanía}

En pleno siglo XXI, las diferencias existentes entre las realidades de los varones y las mujeres siguen siendo lacerantes en términos de inequidad ${ }^{3}$. La desigualdad puede observarse en distintos ámbitos: los escasos puestos de liderazgos ocupados por mu-

gubernamental), la existencia de grupos-objetivo en el origen del problema (se busca modificar u
orientar la conducta de grupos sociales cuyo comportamiento origina el problema que se pretende
resolver), una coherencia institucional (requiere que las decisiones y acciones que se lleven a cabo
estén relacionadas entre sí), la existencia de diversas decisiones y actividades que constituyen un
conjunto de acciones que van más allá de la mera decisión única y específica, un programa de in-
tervenciones (en el que el conjunto de acciones deba incluir decisiones concretas que refieran a la
actuación a implementar), el papel clave de los actores públicos (en el que el conjunto de acciones y
decisiones sólo se consideran políticas sociales cuando quienes las emprenden o toman son actores
públicos) y, finalmente, una naturaleza más o menos obligatoria de las decisiones y actividades,
debido a que las formas de acción son tanto coercitivas como incentivadoras.
3. En referencia a esto, Marcela Lagarde señala que "el mundo contemporáneo se caracteriza por
una organización social de géneros y por una cultura sexista que expresa y recrea la opresión de 
jeres ${ }^{4}$, los menores registros de la presencia femenina en el mercado de trabajo formal directamente relacionados con la llamada feminización de la pobreza ${ }^{5}$, etcétera. Esta situación también es trasladable a la esfera privada del hogar, en la que la desigualdad está enraizada en la distribución inequitativa de las tareas domésticas y de cuidado, arraigadas en estructuras que señalan que son responsabilidad de las mujeres, sólo por su género.

La condición social de la mujer en la actualidad no la ha ubicado muy lejos del modelo patriarcal imperante, a quien se le asigna aún un perfil obligatorio por su naturaleza femenina con una serie de actividades de cuidado de los miembros del grupo. Este trabajo no remunerado parece estar bajo un manto de invisibilidad en el reconocimiento del ámbito económico por la concepción errónea de sólo pertenecer al carácter privado de las relaciones familiares (Antonopoulos y Cos-Montiel, 2007, p. 243).

Alcanzar la igualdad involucra el desmitificar los constructos discursivos que pregonan a los varones como los únicos capaces de resolver asuntos políticos; por lo que, es preciso no sólo erradicar estas naturalizaciones, sino que las mismas políticas tengan entre sus objetivos dispositivos que lo realicen. Para esto, es preciso mencionar a la transversalización de género como mecanismo que permita acometer contra las desigualdades políticas, económicas, sociales que tengan como factor de influencia a los géneros. Esto conllevaría a que el Estado ponga el foco en los procesos de creación y aplicación de las políticas sociales desde una perspectiva de género.

La llamada ciudadanía de las mujeres ha sido desarrollada en contraposición al concepto universal de ciudadanía planteado por varones, para ellos mismos y que excluye a las mujeres. Por décadas, han sido las distintas agrupaciones feministas las que se han opuesto y luchado contra esa masculinización que sufren las mujeres ante la necesidad de ganar más derechos y espacios dentro de un sistema patriarcal -la Conferencia Mundial sobre Población y Desarrollo, la IV Conferencia Mundial sobre la Mujer, son ejemplos claves de las exposiciones sobre esta situación-.

las mujeres y de todas las personas que son diferentes del paradigma social, cultural y político de lo masculino. Se caracteriza, asimismo, por un sistema político, público y privado, de dominio de hombres sobre mujeres" (1990, p.410)

4. En la Argentina, la participación de las mujeres en las cúpulas empresariales es del 15\% (Perfil, 2019).

5. En el primer semestre de 2018, el 37\% de las personas de la población urbana cubierta por la Encuesta Permanente de Hogares (EPH), vivían en hogares donde se identifica a una mujer como la jefa de hogar. Sin embargo, esas personas representaban casi $41 \%$ en los hogares que se encontraban debajo de la línea de pobreza. El porcentaje de personas debajo de la línea de pobreza en hogares con jefa mujer era de casi $30 \%$ mientras que en los hogares donde se identifica a un hombre como jefe ese porcentaje era de alrededor de 26\%” (González Rozada, 2019). 
En esta línea, la politóloga Dietz (2001) plantea que el eje de los cuestionamientos de las feministas debe estar focalizado en quién y cómo se toman las decisiones; interpelar y comprender a la ciudadanía como un bien en sí mismo y como un proceso continuo -en el que es casi imperativa la incorporación activa de la mujer en el mundo público-. Esta última -aunque escasa y a fuerza de leyes de cupo en el caso del ámbito político- ha permitido que muchas constituciones latinoamericanas incluyeran la diversidad junto con la igualdad legal, real, social y de género (Bareiro y Soto, 2017).

En este sentido, esta particularidad que otorga la igualdad debe ser tomada con cautela. La ausencia implícita de ésta en el mito de las oportunidades equitativas nos deja ver aquellos supuestos que niegan la realidad de las inequidades relacionadas a la discriminación sexual, los estereotipos y la subordinación de las mujeres -tanto en ámbitos privados como el hogar como en públicos como el mercado laboral- (Dietz, 2001).

En conjunción a esta postura, la Estrategia de Montevideo para la Implementación de la Agenda Regional de Género en el Marco del Desarrollo Sostenible hacia 2030 (2016) de la CEPAL, plantea que el ejercicio pleno de la ciudadanía femenina estará dado no sólo cuando las mujeres se incorporen activamente a la denominada vida pública, sino cuando puedan contribuir como ciudadanas de pleno derecho. Esto podrá lograrse una vez que las distintas formas de representación política postulen una concepción de desarrollo que incluya a lo privado y no soslaye las diferencias de género.

En esta línea, los economistas Rania Antonopoulos y Francisco Cos-Montiel, -con su interpretación sociopolítica del desarrollo como proceso cultural, político y económico-, analizan esta situación desde la perspectiva de las desigualdades entre mujeres y hombres derivadas de la división sexual del trabajo. Respecto a éstas, plantean que no son cuestionadas, sino que se las comprende como resultado de una organización natural de las funciones sociales. De hecho, exponen que las políticas sociales son formuladas de acuerdo con el modelo de familia nuclear, en el que la mujer toma un rol pasivo dentro de ese desarrollo, debido a que no se la ve más allá de su papel reproductivo y de cuidadora (Antonopoulos y Cos-Montiel, 2007, p. 234).

Ante este análisis, Valenzuela y Mora (2009), presentan a la mujer como un sujeto que convive -o sobrevive- dentro de una pobreza cualitativa y cuantitativamente distinta a la de los hombres. Es que aún, a principios del siglo XXI, los Estados latinoamericanos siguen siendo caracterizados por su naturaleza. Es por esto por lo que se señala al Estado como una institución que (re)produce múltiples dominaciones y discriminaciones de las sociedades latinoamericanas -en especial, aquellas referidas al género- (Bareiro, 1997, p. 3).

El Estado es la institución que cuenta con el poder para que la sociedad cumpla lo que disponen sus dirigentes. Históricamente, bajo sus diversas formas, han sido las mujeres quienes han estado en situaciones de subordinación, en las que el poder era 
ejercido sobre ellas. En este punto es en el que radica la desigualdad mencionada, en la existencia de privilegios que los diversos colectivos tienen en la sociedad y el Estado; en otras palabras, en la comunidad política (Bareiro, 1997).

A pesar del paso del tiempo, esta inequidad poco cambió para las mujeres en función a su rol dentro de la sociedad. De hecho, la antropóloga Feitó (2004) plantea que, si se hace un paralelismo entre "una determinada concepción de la historia de la humanidad, de las relaciones entre hombre y la naturaleza, asumiendo al mismo tiempo un modelo implícito de sociedad considerado como universalmente válido y deseable", se puede inferir que muy lejos está la mujer de alcanzar eso que es "válido y deseable" (p. 5). En otras palabras, esa ciudadanía plena. En conjunción a esto, el concepto de ciudadanía refiere al derecho que tienen las sociedades de intervenir en el poder político y, en simultáneo, a ser intervenidas (Bareiro, 1997).

En este sentido, es preciso traer a colación a la postura de la feminista Chandra Mohanty, respecto al modo en que es construida la mujer como un compuesto cultural e ideológico mediante distintos discursos de representación sobre cómo son las mujeres reales -con sus propias historias-. Es en este punto en el que la autora pone especial énfasis en aquellos discursos que "colonizan de forma discursiva las heterogeneidades materiales e históricas de las vidas de las mujeres en el Tercer Mundo" (Mohanty, 2008, p. 11). Aún así, es importante que en lo relacionado al ámbito laboral se tenga en consideración que "incrementar la participación laboral no debería, sin embargo, ocurrir a todo costo, ni especialmente a costo de las propias mujeres o de las nuevas generaciones. Promover la participación laboral femenina supone reorganizar el orden social, especialmente cuando este no ha sido estructurado considerando la participación laboral femenina" (Cardenas Tomažič y Hein, 2018, p. 167).

\section{El viraje al género de las políticas públicas}

En Latinoamérica, la orientación que tuvieron las políticas sociales desarrolladas en el último cuarto del siglo XX y el primero del siglo XXI, muestra la focalización en determinados grupos sociales como única estrategia posible para alcanzar progresivamente la universalización de la satisfacción de las necesidades básicas (Franco, 1996).

Con la implementación de estas medidas se impulsó a una especie de metamorfosis de la práctica estatal que pretendía la existencia de un equilibrio fiscal, una merma del gasto público y, con esto, mostrar una eficacia en la gestión. Esto se debió a que en los años en los que se hicieron reformas estructurales promercado mutaron drásticamente las maneras de organización y funcionamiento de orden estatal (Acuña, 2011). Lo que hizo que entraran en juego organismos multilaterales dadores de recomen- 
daciones que, más allá de la contextualización físico-temporal situada, propusieron modificaciones en las estructuras estatales ${ }^{6}$ y de las políticas sociales implementadas -o a implementar-.

Con relación a esto, en Argentina, durante los gobiernos de Néstor Kirchner (2003-2007) y Cristina Fernández (2007-2011 y 2011-2015), con el fin de cumplir con el Derecho Familiar de Inclusión Social, se instituyó el Plan Jefes y Jefas de Hogares desocupados. El público objetivo de esta medida fue, tal como su nombre lo indica, los/as jefes/as de hogares desocupados/as con hijos/as menores de 18 años o discapacitados/as. En simultáneo, se instituyó el Programa Familias, cuyo propósito era el de contribuir con el desarrollo de los/as niños/as tanto en el sistema educativo, como en el sanitario; de este modo, a través de esta medida se pretendía evitar situaciones de exclusión en todas aquellas familias ${ }^{7}$ que se encontrasen en condiciones de vulnerabilidad social.

Como complemento, se puso en acción el Programa Emergencia Alimentaria (PEA) para la población que se encontrase en contextos frágiles, ya que se percibían grados de riesgo en el sostenimiento de su subsistencia, por lo que se disponía la provisión de alimentos y otros productos que permitiesen atender necesidades básicas. En este contexto, se implementó también el Programa Emergencia Sanitaria (PES), mediante el cual se garantizaba la provisión de medicamentos y diversos insumos de uso asistencial y hospitalario; al igual que el abastecimiento de Medicamentos Genéricos para la Atención Primaria de la Salud (Bertranou, 2011).

En general, estos Programas de Transferencias Condicionadas de ingreso (PTC) tuvieron como foco el bienestar de niños y niñas, por lo que las receptoras del beneficio fueron las mujeres de estos hogares, ya que se presumía que eran ellas las que velan por la prosperidad de sus hijos e hijas (Bertranou, 2011). En este punto es imprescindible subrayar que el hecho de que estos programas tuvieron como destinatarias a las mujeres no los transformaba en políticas con perspectiva de género.

Otro punto para tener en cuenta fueron las nuevas demandas de acompañamiento estatal, frente a situaciones que a simple vista parecían no resolverse en el marco de la disputa distributiva y que interpelaron al Estado en su capacidad de ofrecer abordajes adecuados y suficientes. Esto se debió a que se abordaron diversos escenarios

6. Para observar -y analizar- estos cambios también es preciso tomar en consideración elementos como el Estado -en cuanto a sus competencias-, la acción -en relación con la intención de ser un acto específico- y la intervención -su capacidad de actuar con el objetivo de cambiar el estado de las cosas dentro de una sociedad-.

7. Respecto a esto, Bertranou (2011) explica que el IDH -Índice de Desarrollo Humano- permitía otorgar subsidios -sujetos a contraprestaciones familiares en salud y escolaridad- a familias en situación de pobreza con hijos menores de 19 años y embarazadas que no reciban ayuda económica del Estado ni asignaciones familiares. 
que combinaron las múltiples consecuencias de encontrarse con "posiciones sociales hostiles y humillantes, desde las cuales no se regresa vía mejoramiento macroeconómico" (Catini, Barchetta y Gómez, 2011, p. 32).

Un punto importante por mencionar es que los PTC no toman en consideración aspectos naturalizados como la distribución desigual del trabajo de cuidado y no remunerado por el género. Al contrario, cuando se les otorga la titularidad a las mujeres, ésta suele ser concedida en su carácter de cuidadora, sin que se incluyan en los componentes del programa aspectos que permitan -o promuevan- una redistribución del trabajo remunerado y no remunerado en el interior de los hogares. En definitiva, hay una ausencia de mecanismos de participación de la voz de las personas beneficiarias y de identificación y atención de las demandas específicas de las mujeres.

En tal sentido, es interesante observar cómo estas diferencias construidas -y jerarquizadas- socialmente, permiten presentar a las relaciones de género como relaciones de poder, en las que los varones tienen un acceso directo a éste; mientras que, en el caso de las mujeres, se encuentra limitado. Esto se debe a que tanto hombres como mujeres viven el poder de un modo distinto, lo que también establece las propias identidades genéricas en función a estas relaciones; situación que se repite cuando se hace la distinción entre lo público y lo privado 8 , lo femenino y lo masculino. Por esto, es importante realizar un análisis que amplíe la visión de lo político ${ }^{9}$, al enmarcar aspectos que aborden temas que se ubican dentro de aquello que es invisible, privado e íntimo.

\section{El Programa Ellas Hacen y su pretendida mirada de género}

Durante la primera gestión presidencial de Cristina Fernández, a través del Ministerio de Desarrollo Social de la Nación se creó el Programa Ingreso Social con trabajo-Argentina Trabaja bajo la Resolución 2476-2010. En el artículo 5 se especifica que la marca registrada Argentina Trabaja es de uso común a los programas del Plan Nacional de Desarrollo local y economía social Manos a la obra. Éste se implementó a partir de 2009.

8. "La división público-privado es una dimensión clave en la conceptualización de las principales estructuras que contribuyen a mantener y reproducir la desigualdad de género, tales como la organización del trabajo, la intimidad y la ciudadanía. Estas estructuras interconectadas están formadas por normas, valores, instituciones y organizaciones que reproducen la desigualdad de género en cada una de estas tres esferas" (Verloo y Lombardo, 2007, p. 28).

9. Para realizar esta afirmación, me apoyo en la postura de Millet, quien planteó en su obra Política sexual (1995) que "lo personal es político", al referirse a la política como un cúmulo de estrategias que también pretende mantener un sistema de dominación patriarcal en ámbitos "privados" como la familia y la sexualidad. Asimismo, eso personal alude a movilizar a las mujeres en colectivo al trasladar lo privado al ámbito de público interés en términos de luchas. 
Estuvo compuesto por diversos programas que tenían como eje el desarrollar la economía social y ahondar en estrategias territoriales. Dentro de éste, en 2013, durante la segunda gestión presidencial de Cristina Fernández, se gesta el Programa Ellas hacen. En marzo de 2013, se incorpora al Programa Ingreso Social con Trabajo bajo la Resolución $\mathrm{N}^{\circ} 2176 / 13$ del Ministerio de Desarrollo Social de la Nación.

El Ellas Hacen fue una política con pretendido enfoque de género que estuvo pensada para aquellas mujeres que se encontrasen en situación de vulnerabilidad socioeconómica y/o eran víctimas de violencia de género. Hasta 2015, las beneficiarias de Ellas Hacen eran 78.377 (Jefatura de Gabinete de Ministros, 2016).

Un punto para destacar es que el Argentina Trabaja se implementó a través de gobiernos municipales y de organizaciones sociales, mientras que el Ellas Hacen se gestionó a través del Ministerio de Desarrollo Social de la Nación, lo que permitió que la medida tuviese un carácter más federal. Tal como lo explica Pacífico (2019), el lanzamiento del Ellas Hacen caracteriza a una época de Argentina, en la que las mujeres se volvieron las principales destinatarias de programas como administradoras de beneficios para los sectores considerados vulnerables socioeconómicamente.

De acuerdo el Ministerio de Desarrollo Social (2014), según los criterios de selección, la población destinataria correspondía a mujeres jefas de hogar con tres o más hijos/as menores de 18 años a cargo, desocupadas, que atravesasen situaciones de violencia.

Por un lado, resulta interesante observar cómo la representación de la "desocupación" excluye al trabajo de cuidado y reproductivo de las mujeres, dejando en claro que desde el Estado no hay un reconocimiento del rol activo de las mujeres en la sociedad. De hecho, esta invisibilización conllevó a diversas tensiones en sus realidades, ya que las beneficiarias del programa se vieron en la obligación de darle una solución a una potencial tercerización de las actividades que realizaban ellas mismas al interior de los hogares (Guerrero, Guerrero y Zangaro 2018).

Por otro lado, en este aspecto, puede observarse que los criterios para acceder al programa también se establecieron en torno a la maternidad. No obstante, en el caso que la mujer aspirante al beneficio no tuviese hijos/as, pero sí fuese víctima de violencia, podía ser exceptuada de este requisito, pero debía contar con un informe social emitido desde el Consejo Nacional de Mujeres. Es decir, la maternidad era un factor importante en el otorgamiento del beneficio. En cuanto al ingreso monetario es clave aclarar que éste era de carácter individual y otorgado en función de las asistencias a las capacitaciones y la conformación de cooperativas.

El objetivo era alcanzar a cien mil mujeres de distintas partes del país -se inscribieron 98.876-, aunque fue la provincia de Buenos Aires la que tuvo mayor cantidad de inscriptas con un 56,4\% del total. A su vez, el Ministerio de Desarrollo Social (2014) destaca que el $83 \%$ de las beneficiarias se encontraban en edad reproductiva al per- 
tenecer a un rango etario 18 a 39 años. Cabe mencionar que una de las características de esta población es su alto índice de desocupación. Esto se refleja en los datos del Ministerio de Trabajo, Empleo y Seguridad Social, al momento de realizarse la primera inscripción en el programa durante el segundo trimestre de 2013, el índice de desocupación de mujeres menores de 29 años correspondía a un 17,4\%, mientras que en el caso de los varones era de un $13,1 \%$.

Tal como lo afirma Isola Zorrozúa (2019) "la población objeto efectivamente conforma "el núcleo duro de la vulnerabilidad y exclusión sociocupacional". Finalmente, según datos del Ministerio de Desarrollo Social, al programa Ellas Hacen ingresaron a nivel nacional 98.876 mujeres, de las cuales el 13,5\% tiene hijos discapacitados, el 20\% declaró situaciones de violencia de género en la preinscripción, y más del 50\% tiene entre 30 y 39 años. Además, el 89,9\% de estas mujeres no ha completado la educación formal y solo el 2,5\% ha finalizado estudios secundarios" (p. 134).

Además, el programa estuvo destinado a la creación de cooperativas por parte de mujeres, con el objetivo de mejorar las condiciones urbanas de los barrios en los que residían. De este modo, a través de esta política, se propuso favorecer tanto a las beneficiarias como a los/as demás habitantes de los asentamientos en los que las mujeres focalizaban su labor, al poder cumplir con el derecho a una vivienda digna para todos y todas.

En relación con esto, el trabajo realizado por las beneficiarias de Ellas Hacen abarcaba desde el reacondicionamiento de lugares públicos -pintura de plazas, limpieza y arreglo de veredas, mantenimiento de espacios verdes, etcétera- a construcciones de hogares y su mantenimiento. Para hacerlo, las mujeres debían capacitarse en diversos oficios y asistir a talleres que les permitiesen alcanzar una mirada empoderadora -economía social, perspectiva de género, etcétera- junto con la posibilidad de terminar sus estudios mediante el Programa FinEs ${ }^{10}$. En este aspecto, cabe aclarar que, al momento de la inscripción, aproximadamente un 20,1\% de las mujeres beneficiarias no había culminado su educación en el Nivel Primario y un 87,9\% no finalizó el Nivel Secundario (Ministerio de Desarrollo Social, 2014).

A través del Ellas Hacen se ingresó a las mujeres beneficiarias a una economía formal, mediante un pago mensual con prestaciones básicas ${ }^{11}$ como obra social y la realización de registros contributivos para el acceso futuro al sistema previsional a través de un monotributo social ${ }^{12}$.

10. Plan destinado a jóvenes y adultos que deseen completar la educación primaria y secundaria. 11. Además, a las cooperativistas se le brindan equipamientos según la labor que realizan y seguros de vida personales y ante tercero a modo de prevención de alguna eventualidad. El monto correspondiente corre por parte del programa.

12. Es una categoría tributaria permanente, creada con el objeto de facilitar y promover la incorporación a la economía formal de aquellas personas en situación de vulnerabilidad que han estado históricamente excluidas de los sistemas impositivos y de los circuitos económicos. 
Mediante este programa, se buscó la generación de empleo a través de organizaciones asociativas -cooperativas- fomentadas y avaladas por el Gobierno, la terminalidad educativa a través del programa $\operatorname{FinEs}^{13}$ y la asistencia en situaciones de violencia de género (Rodríguez Enríquez y Pautassi, 2014). Aquellas beneficiarias del programa percibían los mismos "incentivos de inclusión social" que los/as titulares del Programa Ingreso Social con trabajo-Argentina Trabaja (Arcidiácono y Bermúdez, 2018). Al respecto, es importante contextualizar que "en 2016, el monto máximo a cobrar es de 2.840 pesos, muy por debajo del salario mínimo vital y móvil en Argentina, de 6.060 pesos" (Arcidiáconoy Bermúdez, 2018, p. 4).

Con el Ellas hacen, se pretendía que las mujeres pudiesen fortalecer su autoestima y su autonomía económica a través del estudio, el trabajo en organizaciones asociativas -espacio en el que se generaba compañerismo con mujeres que vivieron situaciones similares- $y$ la posibilidad de conciliar las tareas reproductivas de su hogar con las actividades previstas por el programa (Arcidiácono y Bermúdez, 2018).

\section{Las mujeres y el trabajo en cooperativas}

El Programa Ellas Hacen introdujo al concepto de cooperativas como un factor totalmente novedoso en las políticas destinadas a mujeres. La medida proponía la creación de cooperativas por parte de las beneficiarias como posibilidad de un trabajo autogestivo futuro. Para esto, se les brindaba distintas capacitaciones vinculadas a oficios y a la culminación de niveles educativos incompletos. De este modo, ya no sólo se debía cumplir con ciertos requisitos para la obtención de los beneficios como en los PTC, sino que se proponía una transferencia de ingresos por la realización de trabajos y obtención de capacitaciones.

Como se mencionó previamente, el Ellas Hacen se gestó en el interior del Programa Ingreso Social con trabajo - Argentina Trabaja. El punto clave de esta medida fue la formación de cooperativas laborales, creadas con el aval del Estado. Éstas debían centrarse en el mejoramiento de infraestructura elemental de municipios y comunas por parte de las mujeres beneficiarias del programa que habitaba la zona. En este sentido, el programa se gestaba diferencialmente según el área de acción, ya que, en muchos casos, éste resultaba ser una herramienta para mermar los índices de desocupación e informalidad (Arcidiácono y Bermúdez, 2018).

Como se especificó en el apartado previo, el Ellas Hacen se focalizaba en un grupo específico: mujeres en situación de vulnerabilidad socioeconómica y/o que estuvieran pasando -o vivieron- situaciones de violencia. De este modo, se le agregaba la posibilidad de brindarles herramientas de contención psicológica -se dictaban talleres cuyo eje era la problemática- y laborales -se fomentaba la formación de capital humano-.

13. Resolución N²176/13. 
Este punto es fundamental, ya que si bien se presentó a la política como una medida singular que tomaba en cuenta las realidades de las mujeres en vulnerabilidad; en simultáneo, no se les brindaba soluciones ante la imposibilidad de conciliar la conformación de la cooperativa y las responsabilidades en el ámbito privado del hogar. Es decir, por un lado, se les otorgaba capacitación en diversos oficios, en economía social y la posibilidad de culminar con sus estudios; mientras que, por el otro lado, se perpetuaban las desigualdades al haber una distribución inequitativa de las labores de cuidado y reproductivas en los hogares, provocándose una sobrecarga laboral y de responsabilidades naturalizadas por el género.

En este sentido, hay investigaciones que demostraron que la participación de las mujeres en la armado de cooperativas es beneficioso por poder formarse para participar en el mercado laboral formal y la posibilidad de conciliar con sus labores de cuidado, debido a que la organización está dada en función de las circunstancias de cada una debido a las particularidades autogestivas que estos grupos tienen (OITPNUD, 2009). Este punto es clave debido a los altos índices de desocupación femenina -sin mencionar la subocupación o la presencia en el mercado laboral a través de la informalidad- que, a su vez, les ocasiona peripecias para ingresar al sistema de seguridad social.

Tal como lo afirma Pizarro (2020), "de acuerdo con los datos preliminares obtenidos de la primera fase del Ellas Hacen, el Ministerio de Desarrollo Social de la Nación detalla que, en el contexto del Programa Ingreso Social con Trabajo, la participación de las mujeres ha sido levemente mayor que la de los hombres, con un 54,25\% por parte de las beneficiarias y un 45,75 los varones" (p. 67). Se percibió que, a través de las capacitaciones en liderazgo y economía social, el programa procuró el quiebre con algunos preceptos instaurados como estereotipos masculinos con relación a los campos laborales en los que se pueden desempeñar las mujeres. Con estos cambios intrínsecos de la política se intentó generar una mutación en las estructuras socioculturales que determinan tareas y trabajos específicos en función al género. De hecho, las capacitaciones brindadas abarcaban desde aspectos básicos de economía social a herramientas para defenderse ante situaciones de violencia de género.

Otro dato que destaca el Ministerio de Desarrollo Social de la Nación (2014) es que el $97 \%$ de las beneficiarias del programa declara sentirse con mayor autonomía económica y ser más independiente socialmente a partir de su incorporación al Ellas Hacen. Ante esto, también es importante subrayar que no se aclara el destino real del monto percibido, ya sea para la satisfacción de requerimientos familiares, beneficios de sus hijos/as y/o necesidades personales. 


\section{Talleres de formación con asistencia obligatoria}

La asistencia a los ciclos de formación era obligatoria por parte de las mujeres a las que alcanzaba la medida. Cada taller era financiado por el Estado nacional y trataba de conservar la estructura de la educación formal, efectuándose mediante el Plan FinEs. Éste estuvo destinado no sólo a las beneficiarias del Ellas Hacen, sino a toda aquella persona -jóvenes y adultos sin límite de edad- que desease culminar sus estudios y que no pudo hacerlo por diversas razones.

La cursada consistía en el dictado semanal -dos veces por semana- de materias de contenidos básicos de la currícula educativa nacional, formación que variaba según el nivel que se tuviese, con la posibilidad de su extensión hasta por seis años. Además, en el caso del Ellas Hacen, a las mujeres se les brindó contenidos referidos a los ejes inherentes del cooperativismo. De este modo, el propósito no era sólo inculcar lo pautado en cada cátedra, sino también el presentar al conocimiento como herramienta de cambio; es decir, saberes que les permitan modificar sus realidades.

De hecho, se instauró el Programa Promotores para el Cambio Social, mediante el que se pretendía fomentar la organización y la intervención de distintos actores para el desarrollo de políticas de corte comunitario implementadas por el Ministerio de Desarrollo Social en las distintas comunas. En este sentido, a las cooperativistas que recién se inician en este campo se les brindó a través de este programa "la posibilidad de acompañamiento a través de asistencia técnica y contable para el aumento de sus capacidades como cooperativa de trabajo vinculada a servicios de construcción; considerando esta fase como orientada a generar mayores condiciones para alcanzar la meta de autogestión en el mercado laboral" (Ministerio de Desarrollo Social de la Nación, 2014).

Frente a lo que expresamos en el apartado anterior, el Ellas Hacen pretendía romper los estereotipos de género con la creación de cooperativas de trabajo por parte de mujeres, pero la medida sólo manifestó esta ruptura en el desarrollo de las capacitaciones, no en el diseño del programa, ya que no tuvo en cuenta las singularidades de la división desigual del trabajo reproductivo en los hogares. Otro punto para señalar es que, en referencia al tipo de capacitaciones seleccionadas por las mujeres, la mayor parte de las beneficiarias del Ellas Hacen optaba por aquellas relacionadas al cuidado y tareas feminizadas -atención a adultos mayores, diseño de indumentaria, servicios de limpieza, cocina, entre otros- (Ministerio de Desarrollo Social de la Nación, 2014). Aún así, más allá del tipo de contenidos seleccionados, lo que se pretendió fue formar capital humano femenino que facilitara la inclusión al mercado laboral y la posibilidad de gestar un autoempleo. 
De hecho, tal como lo manifiesta el Ministerio de Desarrollo de la Nación,

la experiencia nos ha mostrado la sinergia de la formación integral para la superación de situaciones de vulnerabilidad extrema y el empoderamiento que ello genera, como ciudadanas en un mundo de derechos y responsabilidades en las cuales poder desplegar sus capacidades. Lo hasta aquí expuesto ha permitido delimitar una línea estratégica específica para la inclusión de mujeres en situación de extrema vulnerabilidad socio ocupacional, la cual será reconocida como ELLAS HACEN (Ministerio de Desarrollo Social de la Nación, 2014).

\section{Manos a la cooperativa}

Una vez que avanzó la capacitación -ésta es continua-, las mujeres debían iniciar con el proceso de formación de la cooperativa propiamente dicha. Ya con los conocimientos asimilados, debían diseñar un proyecto en función a las necesidades que su comuna requiriese. Para esto, las integrantes de la cooperativa en formación debían vivir en un rango próximo o, en el caso de que su proyecto tuviese un objetivo específico -servicios de comida, diseño de indumentaria, etcétera- podían no cumplir con el requisito de proximidad territorial. Una vez que terminasen con el armado de la cooperativa, cada una de ellas recibía un kit de materiales para echar a andar la misma -en muchos casos, los/as capacitadores/as continuaban supervisando las actividades-.

Tal como se mencionó previamente, el Ellas Hacen se encontraba enmarcado en el Programa Argentina Trabaja, por lo que el monto de dinero recibido era similar al resto de los beneficiarios. Éste consistía en un básico de \$1200, al que se le sumaba el presentismo $-\$ 800-y$ un plus por la productividad $-\$ 600-$.

En este sentido, ante la naturalización de la relación del programa con la dualidad mujer-maternidad, se observó el desarrollo de estilos de crianza mediante redes de cuidado familiares que permitían cumplir con lo demandado por el programa para el cobro del beneficio (Sciortino, 2018).

De este modo, el Estado a pesar de resaltar que dicha política tiene una perspectiva de género, se deduce que, en el diseño del programa, no la dejó asentada. De hecho, la modificación respecto al Argentina Trabaja fue sólo designar como público objetivo a mujeres en situación de vulnerabilidad social y/o económica y que hayan atravesado por episodios de violencia de género. Es decir, no se tuvieron en cuenta las particularidades de este grupo poblacional, sólo se concertó como una medida que sirvió como herramienta para la inserción laboral con eje en la solidaridad colectiva a través de la formación de cooperativas de trabajo comunitarias.

Además, otro punto para tener en cuenta es que estas agrupaciones laborales no se diferenciaban de otras en función a sus requisitos para el funcionamiento como tales. Es decir, se les exigía a mujeres -que en muchos casos no habían terminado un 
ciclo básico de formación- el cumplimiento de una tramitación que requiere algo más que una capacitación en principios de cooperativismo, lo que implicaba un esfuerzo mayor por parte de las beneficiarias del Ellas Hacen y más aún si se pretendía que esta iniciativa fuese sostenible en el tiempo.

En este sentido, es oportuno señalar que las condicionalidades impuestas por el programa se encontraban relacionadas con la promoción de la autonomía de las destinatarias, pero los requisitos tenían carácter punitivo. En otras palabras, ¿es posible promover la independencia de las beneficiarias y establecer condicionantes excluyentes para formar parte del plan?

Este aspecto es relevante si se toma en cuenta que las mujeres beneficiarias del programa debían cumplir veinte horas semanales entre capacitaciones, asistencia a establecimientos educativos para su terminalidad educativa, el desarrollo de las actividades que involucraban a las cooperativas, etcétera. Como puede inferirse, las condicionalidades estaban vinculadas a aspectos relacionados con el espacio público y/o el trabajo productivo.

A su vez, el programa parecía alejarse de los modelos tradicionales -y maternalistas-impuestos por los PTCI al proponer en el artículo $4^{\circ}$ de la resolución de su creación deconstruir estereotipos de género a través de las capacitaciones en oficios y la formación en perspectiva de género en derechos de la mujer, la niñez y la familia. Sin embargo, al conservarse el carácter punitivo de los PTCI ante el incumplimiento de los requerimientos exigidos, aspecto característico de este tipo de programas, las beneficiarias se veían condicionadas a crear estrategias que les permitiesen conciliar las tareas reproductivas y de cuidado con las veinte horas de trabajo-capacitación que les demandaba el Ellas Hacen.

Tal como lo afirma Rodríguez Enríquez (2011), la carga de trabajo de las mujeres incrementa con su inclusión a este tipo de programas, ya que hay una sobrecarga de tiempo en las beneficiarias que deben cumplir con lo que se estipula en el plan, mientras se refuerza el rol de cuidadora por la tradicional división sexual del trabajo, que se mantiene estática debido a que no se establecen mecanismos para un potencial cambio de ésta. Al respecto, según datos presentados por el Ministerio de Desarrollo Social (2015), el 93,4\% de las mujeres acreedoras del Ellas Hacen declararon que les fue posible reorganizar el trabajo reproductivo y de cuidado en sus hogares, a pesar de que desde el programa no se estipuló ningún mecanismo de ayuda para que esto fuese posible.

Otro aspecto para tener en consideración es lo referido a la formación, ya que en sí misma no tenía la capacidad de disminuir las discriminaciones presentes en el mercado laboral, debido a que para esto es imperioso que se creen políticas específicas relacionadas a la conciliación y a la justa distribución de las tareas reproductivas y de cuidado. 


\section{Ahora, hacemos futuro}

En 2015, con el inicio de la gestión de Cambiemos ${ }^{14}$, se pausaron a aquellas políticas que tuvieran al cooperativismo como eje, así como a cualquier tipo de actividad comunitaria respaldada por el Estado. Es importante mencionar que, al finalizar la gestión de Cristina Fernández en diciembre de 2015, 81.151 mujeres se encontraban trabajando en aproximadamente 2.90o cooperativas (Ministerio de Desarrollo Social de la Nación, 2014). Con el cambio de la gestión, el programa pasa a denominarse Ellas hacen - Nuevo enfoque, cuyo propósito principal se redujo sólo a brindar facilidades para finalizar los estudios primarios o secundarios, según correspondiese.

A principios de 2018, durante la presidencia de Mauricio Macri, se unifica bajo el nombre "Hacemos futuro" al Plan Argentina Trabaja, Ellas hacen y Desde el barrio. Este nuevo programa estuvo a cargo de la Subsecretaría de Políticas Integradoras de la Secretaría de Economía Social del Ministerio de Desarrollo Social de la Nación ${ }^{15}$. De este modo, se establece como objetivo general del programa "empoderar a las personas o poblaciones en riesgo o situación de vulnerabilidad social, que promuevan su progresiva autonomía económica a través de la terminalidad educativa y cursos y prácticas de formación integral que potencien sus posibilidades de inserción laboral e integración social”"16. Más allá del cambio de denominación y de su unificación con otros programas, las modificaciones también abarcaron las capacitaciones brindadas a los/as beneficiarios/as. Ya no se les capacitaba colectivamente en oficios o formación de cooperativas, sino que los contenidos tomaron un carácter individualista, con influencias de la autoayuda y tips de autosuperación.

Es así como se renombra al Ellas Hacen como subprograma Hacemos futuro juntas, cuyo primer objetivo es "Establecer la estrategia y las herramientas necesarias para la implementación de la línea programa Ellas Hacen” ${ }^{17}$, pero sin determinar objetivos propios ${ }^{18}$ que fomenten una perspectiva de género desarrolladora y superadora a la política antecesora ${ }^{19}$.

14. Cambiemos es un partido político argentino inscripto para competir en las elecciones nacionales del año 2015. Bajo este nombre se realizó una coalición entre la Unión Cívica Radical, Coalición Cívica Ari, entre otros. Mauricio Macri fue quien presidía el partido y quien ganó las elecciones del periodo 2015-2019.

15. Resolución de la Secretaría de Economía Social N 151, Art. 2, República Argentina, 2018.

16. Resolución del Ministerio de Desarrollo Social de la Nación, N96, Art. 2, República Argentina, 2018.

17. Resolución del Ministerio de Desarrollo Social de la Nación Nº 1274 -E, Anexo V, República Argentina, 2017.

18. Resolución 1274-E/2017 del Ministerio de Desarrollo Social de la Nación, República Argentina, 2017.

19. Resolución N²176/13 del Ministerio de Desarrollo Social de la Nación, República Argentina, 2017. 
De este modo, ya no sólo se desdibujó ese pretendido enfoque de género que tenía el Programa Ellas Hacen, sino que con esta unificación de políticas se invisibilizó por completo a la mujer, sus particularidades y la posibilidad de salir de contextos de violencia física y psicológica. Es decir, se disiparon los espacios de contención y crecimiento de mujeres que pensaron que podrían contar con el respaldo de un Estado que había empezado a visibilizarlas, a valorarlas.

En tanto, el Programa Hacemos Futuro expresa a la transferencia de ingreso como un subsidio personal por capacitación, que, a su vez, permite el acceso al Monotributo Social y seguro (Arcidiácono y Bermúdez, 2018, p. 4). En cuanto a la diferenciación, más allá de su denominación, los objetivos del programa son muy similares, ya que se pretende que mediante la finalización de los ciclos curriculares básicos -a través del Plan FinEs-, se alcance cierta autonomía económica al lograrse una posibilidad de acceder al mercado laboral. En caso de la obtención de un trabajo formal, las/ os titulares continúan siendo parte del programa, pero con la cesación de pago del subsidio por un año.

Al dar por finalizado al Programa Ellas Hacen se continuó con la lógica patriarcal en la que las mujeres son invisibilizadas a través de la generalización, mediante la que se pretende encuadrarlas en requisitos que no tienen en consideración las singularidades del cuidado y tareas reproductivas que ellas realizan, entre otros factores. De hecho, son estas singularidades las que las ha mantenido alejadas -muchas veces, excluidas- del mercado laboral y de un posible acceso al sistema de seguridad social.

\section{Consideraciones finales}

El Programa Ellas Hacen permitió poner en discusión el papel de las mujeres por parte del Estado, al vérselas sólo como madres y cuidadoras, pero sin tener en cuenta las peripecias que estos roles conllevan. Esta política surgió como respuesta a la presencia intermitente de las mujeres en el mercado laboral formal, la subocupación femenina, las situaciones de pobreza en hogares en los que, en muchos casos, el trabajo precarizado de la mujer era el único sustento.

De este modo, el Ellas Hacen permitió la formación de capital humano a través de la terminalidad educativa y la especialización en oficios, que en un futuro les permitiría proyectar la creación de cooperativas de trabajo autogestionadas por las beneficiarias. A pesar de esto, se puede discurrir en que, a pesar de tener el apoyo estatal, les resultaba muy dificultoso a las mujeres ser autogestoras sin poseer la formación densa y definida que este tipo de proyectos requiere.

Otro punto en cuestión es que, siendo una política con pretendido enfoque de género, se les exigía a sus beneficiarias el cumplimiento de ítems como presentismo y/o producción, sin vislumbrar factores como el cuidado de personas a su cargo. De hecho, ésta es una gran falla por parte del Estado argentino, que carece de servicios 
de cuidado gratuitos para madres que deseen desempeñarse laboralmente y no pueden hacerlo por no tener esta posibilidad. Quizás el Programa Ellas Hacen hubiese sido una política con enfoque de género si este tipo de servicio hubiese estado en el diseño de la misma medida, ya que es un punto clave para lograr el desarrollo de la autonomía femenina.

En efecto, resaltar las particularidades de las mujeres como cuidadoras podría haber sido el puntapié para crear políticas de cuidado que rediman estas necesidades específicas, con la potencialidad de ser monitoreadas y evaluar cuál sería el impacto de estas medidas en la vida de las mujeres de los distintos sectores.

En definitiva, el Programa Ellas Hacen fue una política icónica en Argentina, que dejó en manifiesto la mirada patriarcal que el Estado tuvo en su diseño y desarrollo, a pesar de ser presentada como una medida disruptiva para la época. En este sentido, es clave, entonces, poder reconocer las perspectivas con las que se rigen las agendas políticas de los gobiernos para observar las prioridades y las acciones que se establecen en los planes de acción gubernamentales. Esto, permitirá comprender los diseños de las políticas públicas y relacionarlos con el contexto sociopolítico en el que se desarrolla.

\section{Referencias}

Acuña, Carlos (2011). ¿Cuánto importan las instituciones? Gobierno, Estado y actores en la política argentina. Serie Estado y Política, Siglo XXI, Buenos Aires, Argentina.

Antonopoulos, Rania y Francisco Cos-Montiel (2007). Estado, diferencia, diversidad: buscando un camino con mayor democracia e igualdad de género. Lima, Sede PNUD.

Arcidiácono, Pilar y Ángeles Bermúdez (2018). ¿Cooperativismo como oportunidad perdida? Problemas estructurales y coyunturales del cooperativismo bajo programas. Ciudadanías, 2, 83-111.

Arcidiácono, Pilar y Ángeles Bermúdez (2018). Ellas Hacen. Programas sociales y exigencias a las mujeres en Argentina. Revista Estudos Feministas, 26, 1-16.

Bareiro, Line (1997). Construcción femenina de ciudadanía. En Bareiro, L. y Soto, C. (Ed) Ciudadanas: una memoria inconstante. Venezuela, Nueva sociedad.

Bareiro, Line y Clyde Soto (2016). Igualdad de género mediante Políticas Públicas en estados inclusivos en el marco de la agenda 2030. Borrador Documento de Insumo. Montevideo, Uruguay.

Bertranou, Fabio (2011) Encrucijadas en la seguridad social argentina: reformas, cobertura y desafios para el sistema de pensiones, CEPAL y Oficina Internacional del Trabajo, Buenos Aires, Argentina. 
Cardenas Tomažič Ana y Kerstin Hein (2018). La mujer flexible. Puntos de inflexión biográficos y conducción de vida cotidiana de trabajadoras-madres. En A. Cardenas Tomažič y A. M. Yevenes Ramirez (Comp.) Mujer(es), familia(s) y trabajo(s). Un debate internacional. Buenos Aires, Editorial Teseo.

Catini, Alejandra, Judith Barchetta y Ana Gómez (2011). El análisis y las intervenciones sobre la pobreza: la complejidad como desafío, La Plata, Argentina.

Dietz, Mary (2001). El contexto es lo que cuenta: feminismo y teorías de la ciudadanía. En M. Lamas (Comp.), Ciudadanía y feminismo (pp. 105-130). México, Metis, Estudios Culturales.

Feitó, María Carolina (2004). Antropología y Desarrollo Rural: Contribuciones del Abordaje Etnográfico a los Procesos de Producción e Implementación de Políticas. Avá. Revista de Antropología, 6, 1-26.

Franco, Rolando (1996). Los paradigmas de la política social en América Latina, Revista de la CEPAL, 58, CEPAL, Santiago de Chile. Recuperado de <https://repositorio.cepal.org/handle/11362/12009>.

González Rozada, Martín (2019). Evolución histórica de la desigualdad de ingresos y de la incidencia de la pobreza en Argentina. Foco Económico. Recuperado de https:// focoeconomico.org/2019/04/10/evolucion-historica-de-la-desigualdad-de-ingresos-y-de-la-incidencia-de-la-pobreza-en-argentina/.

Guerrero, Romina, Gabriela Guerrero y Marcela Zangaro (2018). Reflexiones Sobre El Trabajo De Cuidados: Análisis De La Experiencia Del Programa "Ellas Hacen". Mediacoes, 23, 195-221.

Isola Zorrozúa, Florencia (2019). Trayectorias de cuidados en el programa social Ellas Hacen. En Los derroteros del cuidado. Compilado por G. Nelba Guerrero, K. Ramacciotti y M. Zangaro, (pp. 138-155). Buenos Aires, Argentina: Universidad Nacional de Quilmes.

Jefatura de Gabinete de Ministros (2016). Memoria detallada del estado de la Nación 2016. Buenos Aires: Jefatura de Gabinete de Ministros. Presidencia de la Nación.

Lagarde, Marcela (1990). Cautiverio de las mujeres: madres, esposas, monjas, putas, presas y locas. México, UNAM.

Millet, Kate (1995). Política sexual. Madrid: Cátedra.

Ministerio de Desarrollo Social de la Nación (2014). Primer Informe. Antecedentes, creación y primera etapa de Ellas Hacen. Buenos Aires, Argentina, Presidencia de la Nación. Recuperado de http://www.desarrollosocial.gob.ar/Uploads/i1/1er\%20 informe,\%20antecedentes\%20creacion\% 20y\%201er\%20etapa.pdf. 
Mohanty, Chandra (2008). Bajo los ojos de Occidente. Academia feminista y discurso colonial. En L. Suárez Navaz y A. Hernández (Ed). Descolonizando el Feminismo: Teorías y prácticas desde los márgenes (pp. 8-24). Madrid, Ed. Cátedra.

OIT-PNUD (2009). Trabajo y familia: Hacia nuevas formas de conciliación con corresponsabilidad social. Santiago de Chile, OIT-PNUD.

Pacífico, Florencia (2019). Más allá del programa. Etnografía de experiencias cotidianas de mujeres titulares del Ellas Hacen. Papeles de Trabajo 37, 77-111.

Pizarro, Tatiana Marisel (2020). Representaciones sobre el trabajo femenino en la política pública. El caso Ellas Hacen (2013-2019). En J. C. Suárez, N. Martínez y P. Panarese (Eds). Cartografía de los micromachismos: dinámica y violencia simbólica (pp. 55-75). Madrid, Editorial Dykinson.

Resolución de la Secretaría de Economía Social N 151, Art. 2, República Argentina, 2018.

Resolución del Ministerio de Desarrollo Social de la Nación, N96, Art. 2, República Argentina, 2018.

Resolución del Ministerio de Desarrollo Social de la Nación Nº 1274 -E, Anexo V, República Argentina, 2017.

Resolución 1274-E/2017 del Ministerio de Desarrollo Social de la Nación, República Argentina, 2017.

Resolución N²176/13 del Ministerio de Desarrollo Social de la Nación, República Argentina, 2017.

Rodríguez Enríquez, Corina (2011). Programas de Transferencias Condicionadas de Ingreso e Igualdad de Género. ¿Por dónde anda América Latina?, Serie Mujer y Desarrollo 109, Santiago, Chile.

Rodríguez Enríquez, Corina y Laura Pautassi (2014). La organización social del cuidado de niños y niñas. Buenos Aires, Argentina.

Sciortino, Silvana (2018). Una etnografía sobre arreglos familiares, leonas y mujeres superpoderosas. Prácticas compartidas entre las titulares del «Ellas Hacen». Cuadernos de Antropología Social, 55- 71.

Subirats, Joan (2000). Análisis de Politicas públicas y eficacia de la administración. Ministerio para las administraciones públicas, Madrid.

Valenzuela, María Elena y Claudia Mora (2009). Esfuerzos concertados para la revalorización del trabajo doméstico remunerado. En Valenzuela, M. y Mora, C. (Ed). Trabajo doméstico: un largo camino hacia el trabajo decente (pp. 285-301). Santiago, OIT. 
Verloo, Mieke y Emanuela Lombardo (2007). Contested Gender Equality and Policy Variety in Europe: Introducing a Critical Frame Analysis Approach. En M. Verloo (Ed.). Multiple Meanings of Gender Equality. A Critical Frame Analysis of Gender Policies in Europe (pp. 21-46). Budapest, CEU Press.

\section{Sobre la autora}

Tatiana Marisel Pizarro es Doctora en Ciencias Sociales en la Universidad Nacional de Cuyo (2020), Becaria Posdoctoral del Consejo Nacional de Investigaciones Científicas y Técnicas e investigadora del Instituto de Investigaciones Socioeconómicas de la Universidad Nacional de San Juan. Sus líneas de investigación son políticas públicas, estudios interdisciplinarios de género y análisis de discursos. Correo Electrónico: tatianamariselpizarro@gmail.com. D https://orcid.org/0000-0002-3701-5156 


\title{
CUHSO
}

Fundada en 1984, la revista CUHSO es una de las publicaciones periódicas más antiguas en ciencias sociales y humanidades del sur de Chile. Con una periodicidad semestral, recibe todo el año trabajos inéditos de las distintas disciplinas de las ciencias sociales y las humanidades especializadas en el estudio y comprensión de la diversidad sociocultural, especialmente de las sociedades latinoamericanas y sus tensiones producto de la herencia colonial, la modernidad y la globalización. En este sentido, la revista valora tanto el rigor como la pluralidad teórica, epistemológica y metodológica de los trabajos.

\author{
EDITOR \\ Matthias Gloël \\ COORDINADORA EDITORIAL \\ Claudia Campos Letelier \\ CORRECTOR DE ESTILO Y DISEÑADOR \\ Ediciones Silsag \\ TRADUCTOR, CORRECTOR LENGUA INGLESA \\ Aurora Sambolin Santiago \\ SITIO WEB \\ cuhso.uct.cl \\ E-MAIL \\ cuhso@uct.cl \\ LICENCIA DE ESTE ARTÍCULO \\ Creative Commons Atribución Compartir Igual 4.0 Internacional
}

